\section{Optimization of the tartrate-resistant acid phosphatase detection by histochemical method}

M.J. Galvão, ${ }^{1}$ A.R. Santos, ${ }^{1}$ M.D. Ribeiro, ${ }^{2}$

A. Ferreira, ${ }^{1,3}$ F. Nolasco ${ }^{1,3}$

'Laboratório de Morfologia Renal, Serviço

de Nefrologia, Hospital de Curry Cabral

'Serviço de Anatomia Patológica,

Hospital Distrital de Chaves

${ }^{3}$ Faculdade de Ciências Médicas,

Universidade Nova de Lisboa, Portugal

\section{Abstract}

According to the new kidney disease improving global outcomes (KDIGO) guidelines, the term of renal osteodystrophy, should be used exclusively in reference to the invasive diagnosis of bone abnormalities. Due to the low sensitivity and specificity of biochemical serum markers of bone remodelling, the performance of bone biopsies is highly stimulated in dialysis patients and after kidney transplantation. The tartrate-resistant acid phosphatase (TRACP) is an iso-enzyme of the group of acid phosphatases, which is highly expressed by activated osteoclasts and macrophages. TRACP in osteoclasts is in intracytoplasmic vesicles that transport the products of bone matrix degradation. Being present in activated osteoclasts, the identification of this enzyme by histochemistry in undecalcified bone biopsies is an excellent method to quantify the resorption of bone. Since it is an enzymatic histochemical method for a thermolabile enzyme, the temperature at which it is performed is particularly relevant. This study aimed to determine the optimal temperature for identification of TRACP in activated osteoclasts in undecalcified bone biopsies embedded in methylmethacrylate. We selected 10 cases of undecalcified bone biopsies from hemodialysis patients with the diagnosis of secondary hyperparathyroidism. Sections of $5 \mu \mathrm{m}$ were stained to identify TRACP at different incubation temperatures $\left(37^{\circ}, 45^{\circ}, 60^{\circ}, 70^{\circ}\right.$ and $\left.80^{\circ} \mathrm{C}\right)$ for $30 \mathrm{~min}$. Activated osteoclasts stained red and trabecular bone (mineralized bone) was contrasted with toluidine blue. This approach also increased the visibility of the trabecular bone resorption areas (Howship lacunae). Unlike what is suggested in the literature and in several international protocols, we found that the best results were obtained with temperatures between $60^{\circ} \mathrm{C}$ and $70^{\circ} \mathrm{C}$. For technical reasons and according to the results of the present study, we recommended that, for an incubation time of $30 \mathrm{~min}$, the reaction should be carried out at $60^{\circ} \mathrm{C}$. As active osteoclasts are usually scarce in a bone section, the standardization of the histochemistry method is of great relevance, to optimize the identification of these cells and increase the accuracy of the histomosphometric results. Our results, allowing an increase in osteoclasts contrast, also support the use of semi-automatic histomorphometric measurements.

\section{Introduction}

Uremic patients present significant morbidity of bone and mineral metabolism, which has been associated with osteoporosis, bone fractures, vascular calcification, and increased mortality. Some abnormalities can be found since early stages of chronic renal failure (CKD), but they are particularly relevant in dialysis patients and after kidney transplantation. Contrarily to what observed in general population, in uremic patients the biochemical markers of bone remodelling have a low specificity and sensitivity in the diagnosis of bone turnover. Many of these patients are anuric or with severe CKD, not allowing the use of urine markers of bone remodelling.

For these reasons, the undecalcified bone biopsy is the method of choice for evaluation of renal osteodystrophy, ${ }^{1,2,3}$ allowing the characterization of bone remodelling, osteoide mineralization and bone volume (cortical and trabecular) as defined by the new "turnover, mineralization, volume" (TMV) classification of kidney disease improving global outcomes (KDIG0). ${ }^{4}$

Resorption activity is usually much more difficult to quantify than all the other bone histomorphometric parameters, because osteoclasts are usually scarce in a bone section. For this reason, the standardization of a histochemistry method to optimize the identification of active osteoclasts would be highly relevant. This was the objective of the present investigation, based on the expression of acid phosphatase by active osteoclasts.

Tartrate-resistant acid phosphatase (TRACP) belongs to the acid phosphatases (AcP) - EC 3.1.3.2. The catalytic activity of these enzymes target phosphoesters in acidic environments. ${ }^{5}$ TRACP is a cationic ferric glycoprotein with a molecular mass of about 35 $\mathrm{kDa}$ and a monomeric peptide structure. ${ }^{6}$ TRACP expression is found in cells of the mononuclear phagocyte system, most abundantly in bone-resorbing osteoclasts, alveolar macrophages and dendritic cells. ${ }^{5}$

TRACP activity was first detected in the ruffled border membrane. Later immunohistochemical studies detected TRACP in intracellu-
Correspondence: Maria João Galvão, Hospital de Curry Cabral, Serviço de Nefrologia, Laboratório de Morfologia Renal, Rua da Beneficência, No8, 1069-639 Lisboa, Portugal.

Tel. +35.1.217924229 - Fax: +35.1.217924319.

E-mail: mariajoaolopesgalvao@hotmail.com

Key words: tartrate-resistant acid phosphatase, osteoclasts, bone resorption, histochemistry, bone biopsy, renal osteodystrophy.

Contributions: all authors contributed to the planning of this study, experimental procedures, analysis of data obtained and in the writing of this paper. All authors approve the final version.

Conflict of interest: the authors declare no potential conflict of interest.

Received for publication: 2 August 2010. Accepted for publication: 9 November 2010.

This work is licensed under a Creative Commons Attribution 3.0 License (by-nc 3.0).

○C Copyright M.J. Galvão et al., 2011

Licensee PAGEPress, Italy

European Journal of Histochemistry 2011; 55:e1 doi:10.4081/ejh.2011.e1

lar vesicles but not in the ruffled border region. However, contradictory results were found using immunogold technique, showing strong staining in both structures. There is an actual agreement that TRACP is mainly produced and secreted from osteoclasts during bone resorption. $^{5}$

TRACP has a binuclear iron center and, like other metallo-proteins, catalyzes the formation of reactive oxygen species (ROS), compounds with the ability to destroy peptide bonds.? These compounds are secreted by osteoclasts participating in the process of bone matrix degradation. $^{8}$

In general, ROS have been shown to stimulate osteoclastic bone resorption, both in vivo and in vitro, and enhance recruitment of osteoclasts. $^{5}$

Both ROS generation and AcP activity of TRACP use the redox active iron for the catalysis. However, the AcP activity has an acidic $\mathrm{pH}$ optimum and ROS are generated in a neutral PH. Single amino acid TRACP mutants that are completely inactive as phosphatases maintain the ability to produce ROS, suggesting that the two activities are functionally independent. ${ }^{5}$ Beck et al. ${ }^{9}$ demonstrated that the AcP activity of TRACP is inhibited by the substrate of ROS generating activity and vice versa which confirm the use of the same active site.

TRACP is also responsible for dephosphorylation of bone phosphoproteins like osteopontin, a protein involved in binding osteoclasts to the bone trabecula. ${ }^{10}$ This action suggests that 
TRAP is also involved in the regulation of bone resorption. 0steoclasts, also through the secretion of hydrochloric acid and proteases, degrade bone matrix, mainly through the breakdown of links of collagen type $\mathrm{I}^{11}$ The products of degradation of bone matrix are phagocytised and transported intracellularly by intracytoplasmic vesicles. TRACP is found in these vesicles suggesting that the fragmentation of the material occurs, not only extracellularly in Howship lacunae, but also intracellularly. ${ }^{7}$

The role of TRACP in bone metabolism has been elucidated by TRACP knock-out and over expressing mouse models. Both models exhibit a specific bone phenotype. Mice lacking TRACP have mild osteopetrosis resultant of defective bone resorption. ${ }^{12}$ Mice over expressing TRACP have increased bone resorption and formation resulting in mild osteoporosis. ${ }^{13}$

All these characteristics of TRACP make this enzyme an important factor in the diagnosis of renal osteodystrophy.

The enzymatic method used in this study belongs to the category of intramolecular rearrangements. In this category, an initial colourless soluble substrate, after hydrolysis, undergoes a molecular rearrangement producing an insoluble coloured final product. The rearragement is due to the reaction of the substrate with a diazonium salt (like pararosaniline) forming a chromophore azo group allowing the detection of the reaction. ${ }^{14}$

Bancroft and Stevens ${ }^{14}$ suggested a method similar to that used in this study. The main differences are the absences of tartaric acid and manganese sulphate in the incubation solution, and the referred temperature at $37^{\circ} \mathrm{C}$.

The first publication of this method applied specifically to undecalcified bone samples was by Evans et al. ${ }^{15}$ The method described differs from ours, in the use of tartaric acid and incubation time $(1 \mathrm{~h})$. Also, the referred temperature was $37^{\circ} \mathrm{C}$. TRACP is resistant to tartrate while other enzymes present in the bone (which hydrolyze the same substrate) are not. ${ }^{16}$ The manganese has an activator effect on TRACP. ${ }^{17}$ The study of undecalcified bone biopsies is done through various histochemical methods that allow the evaluation of parameters such as the trabecular and cortical areas/volumes, bone mineralization and osteoid tissue, the trabecular surface with deposits of aluminum and/or iron, the osteoblastic surface and activated osteoclasts. ${ }^{18}$ Activated osteoclasts arise in small quantity in a normal bone biopsy, and they are even absent in many cases of adynamic bone, which makes their identification particularly difficult. The quantification of activated osteoclasts enables the determination of the degree of bone resorption,,$^{19}$ becoming thus essential for the study of bone metabolism.

The method used in this study was opti- mized in the Laboratório de Morfologia Renal of the Nephrology Service, Hospital Curry Cabral, Lisboa, Portugal for the study of undecalcified bone biopsy embebbed in methylmethacrylate (MMA). This study aimed at determining the optimal temperature (at a fixed incubation time of $30 \mathrm{~min}$ ) for the identification of TRACP in osteoclasts in undecalcified bone biopsies and embedded in MMA.

\section{Materials and Methods}

Undecalcified bone biopsies from 10 uremic patients with the diagnosis of secondary hyperparathyroidism (due to the higher number of activated osteoclasts) were selected. ${ }^{20}$

These biopsies were processed according to the protocol of the Laboratório de Morfologia Renal of the Nephrology Service, Hospital Curry Cabral: ${ }^{21}$

fixation $-70 \%$ alcohol -8 days at room temperature (RT);

dehydration - $96 \%$ alcohol $-24 \mathrm{~h}$ at RT, $100 \%$ alcohol - $24 \mathrm{~h}$ at RT, acetone -3 days at RT; impregnation - impregnation solution -3 days at $-20^{\circ} \mathrm{C}$;

polymerization - inclusion solution - 24 to 48 $h$ at RT.

Both impregnation and inclusion solutions had the same basis.

\section{Solution A}

MMA (Merck, Darmstadt, Germany); sodium hydroxide (BDH, Poole, England) $0.5 \mathrm{~N}$; add slowly in a proportion of 2MMA:1Sodium hydroxide, well mixed and let it rest a few min until the solution presented two phases. Then, we reject the brown part in the bottom and kept the colourless liquid. This procedure was repeated until the solution only presented one phase, then stored at $-20^{\circ} \mathrm{C}$ for 24 hs.; we filtered the solution and stored again at $-20^{\circ} \mathrm{C}$.

\section{Solution B}

Solution A - $300 \mathrm{~mL}$; benzoyl peroxide (Merck) $-2 \mathrm{~g}$; Stored at $-20^{\circ} \mathrm{C}$ for $24 \mathrm{~h}$, then filtered and stored again at $-20^{\circ} \mathrm{C}$.

\section{Solution C}

Solution B - $300 \mathrm{~mL}$; 2-hydroximethylmethacrylate (Merck) - $150 \mathrm{~mL}$; polyethylenglycol 400 (Merck) - $50 \mathrm{~mL}$.

\section{Impregnation solution}

Solution C - $15 \mathrm{~mL}$; 5\% NN-dimethylaniline (BDH) in Isopropyl alcohol (Merck) $-90 \mu \mathrm{L}$.

\section{Inclusion solution}

Solution C - $15 \mathrm{~mL}$; 5\% NN-dimethylaniline (BDH) in isopropyl alcohol (Merck) $-120 \mu \mathrm{L}$.

From each biopsy five sections of $5 \mu \mathrm{m}$ were performed. The sections were cut in a semi- automatic microtome (Leica RM-2155, Wetzlar, Germany) equipped with a tungsten blade, profile d (Reichert-Jung, NY, USA). The sections were placed on slides with Haupt's gelatine. Haupt's gelatine was prepared by dissolving $1 \mathrm{~g}$ of gelatine (Merck) in $100 \mathrm{~mL}$ of distilled water at $55^{\circ} \mathrm{C}$ and, after cooling, $15 \mathrm{~mL}$ of glycerol (Merck) and $2 \mathrm{~g}$ of phenol (Merck) were added.

The slides were involved in a polyethylene film and placed in a press in the incubator at $60^{\circ} \mathrm{C}$ for two days.

The MMA was not dissolved prior to staining the sections.

\section{Method of TRACP detection}

The sections of each biopsy were incubated at different temperatures $\left(37^{\circ} \mathrm{C}, 45^{\circ} \mathrm{C}, 60^{\circ} \mathrm{C}\right.$, $70^{\circ} \mathrm{C}$ and $80^{\circ} \mathrm{C}$ ) with the incubation solution in a humid chamber for $30 \mathrm{~min}$.

Incubation solution: i) pararosaniline (Merck) 4\% (in hydrochloric acid 20\%) - $1 \mathrm{~mL}$; ii) sodium nitrite (Merck) $3,75 \%$ - $3 \mathrm{~mL}$; iii) AS-TR naphthol phosphate (Sigma-Aldrich, Missouri, USA) $1,6 \%$ in dimethylformamide (Merck) - $2 \mathrm{~mL}$; iv) $0.1 \mathrm{M}$ acetate buffer pH5 $20 \mathrm{~mL}$; sodium acetate $0.1 \mathrm{M}-70 \mathrm{~mL}$; acetic acid $0.6 \%$ - $30 \mathrm{~mL}$ (always confirm the $\mathrm{pH}$ before use); v) manganese sulphate (Merck) 10\% - 6 drops; vi) tartaric acid (Sigma-Aldrich) - $260 \mathrm{mg}$.

The solution has to be filtered before adding the tartaric acid.

This solution can be stored at $-20^{\circ} \mathrm{C}$ for several months. Before use, the solution must be kept at room temperature and filtered.

After washing with distilled water and dried with filter paper the contrast was obtained with toluidine blue (Sigma-Aldrich). After drying with filter paper, the slides were placed in xylene and mounted with Entellan (Merck).

The slides were observed at an optical microscope at the magnifications of $375 \mathrm{x}$ by two of the authors without the knowledge of case or temperature of the staining.

The slides were classified as: ++ intensively stained, + stained, 0 unstained, +- stained with artefacts, 0 - unstained with artefacts. Cases were the sections fell of the slides were considered as NA (not applicable).

The study was approved by the ethical committee of the Hospital Curry Cabral, and each patient signed an informed consent form.

\section{Results}

As expected, activated osteoclasts stained red (allowing to distinguishing certain cellular characteristics such as multinucleation) and trabecular bone stained blue. Activated osteoclasts stained with a red colour of high inten- 
sivity, which allows the performance of semiautomatic bone histomorphometry.

Table 1 shows that at $37^{\circ} \mathrm{C}$ and $45^{\circ} \mathrm{C}$ there was in all cases, an absence of staining of osteoclasts. At $60^{\circ} \mathrm{C}$ and $70^{\circ} \mathrm{C}$ the results were similar. These two temperatures provided the best results.

Figures $1\left(60^{\circ} \mathrm{C}\right)$ and Figure $2\left(70^{\circ} \mathrm{C}\right)$ show the strong staining of osteoclasts obtained at both temperatures. At $80^{\circ} \mathrm{C}$ the results were variable. There were cases in which the sections fell from the slides. In other cases, at this high temperature, it was observed a constant presence of artefacts such as precipitation of crystals with a heterogeneous staining that included some pale areas in the osteoclasts cytoplasm, as shown in Figure 3.

\section{Discussion}

Relevant bone abnormalities can be found since early stages of chronic renal failure (CKD). They are particularly relevant in dialysis patients and after kidney transplantation. The non-invasive diagnosis of bone turnover,

Table 1. Staining classification.

\begin{tabular}{lccccc} 
Case \# & $37^{\circ} \mathrm{C}$ & $45^{\circ} \mathrm{C}$ & $60^{\circ} \mathrm{C}$ & $70^{\circ} \mathrm{C}$ & $80^{\circ} \mathrm{C}$ \\
1 & 0 & 0 & ++ & + & $0-$ \\
2 & 0 & 0 & ++ & ++ & +- \\
\hline 3 & 0 & 0 & + & ++ & NA \\
4 & 0 & 0 & ++ & ++ & +- \\
\hline 5 & 0 & 0 & ++ & ++ & $0-$ \\
6 & 0 & 0 & ++ & + & NA \\
\hline 7 & 0 & 0 & + & ++ & $0-$ \\
8 & 0 & 0 & ++ & + & $0-$ \\
\hline 9 & 0 & 0 & + & ++ & +- \\
10 & 0 & 0 & ++ & ++ & NA \\
\hline
\end{tabular}

++ , intensively stained; +, stained; 0 , unstained; +-, stained with artefacts; 0 -, unstained with artefacts; NA, not applicable.

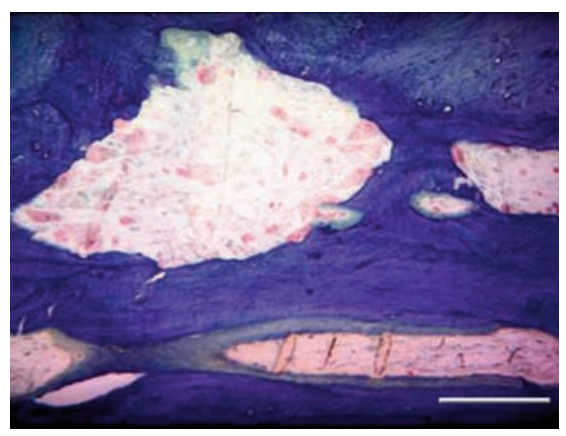

Figure 1. TRACP staining, incubation at $60^{\circ} \mathrm{C}$. All osteoclasts are stained red and no artifact is observed. Scale bar: $\mathbf{4} \boldsymbol{\mu m}$. presents relevant limitations (lack of specificity and sensitivity). For these reason, the major objective of our investigation was to determine the bone formation and resorption, the bone mineralization and bone volume in un-decalcified bone biopsies (according to the TMV classification). The bone resorption activity is very difficult to evaluate and quantify due to the imbalance with bone formation. The measurement of active resorption surface, number of osteoclasts and eroded surface can be optimized by a specific staining for osteoclasts, as TRACP.

The staining results at different temperatures of $37^{\circ} \mathrm{C}, 45^{\circ} \mathrm{C}, 60^{\circ} \mathrm{C}$ and $70^{\circ} \mathrm{C}$ were homogeneous.

At $80^{\circ} \mathrm{C}$ there was no homogeneity, probably because this temperature is close to the denaturation temperature of TRACP. Also, this temperature seems to be too aggressive, since in three cases occurred the loss of sections.

The best results were obtained at $60^{\circ} \mathrm{C}$ and $70^{\circ} \mathrm{C}$. However, at $70^{\circ} \mathrm{C}$ in cases where sections are not well adherent may exist the risk of falling from slides.

Compared with the method described by Bancroft and Stevens,${ }^{14}$ where the recommended temperature is $37^{\circ} \mathrm{C}$ but with a very wide time range from 15 to $60 \mathrm{~min}$, our approach and results are clearly different. In fact, we

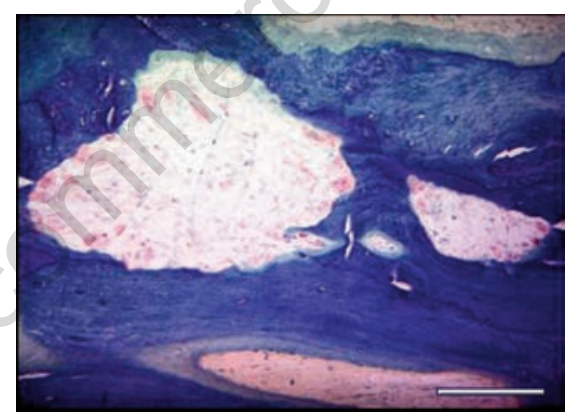

Figure 2. TRACP staining, incubation at $70^{\circ} \mathrm{C}$. All osteoclasts are stained red and no artifact is observed. Scale bar: $4 \mu \mathrm{m}$.

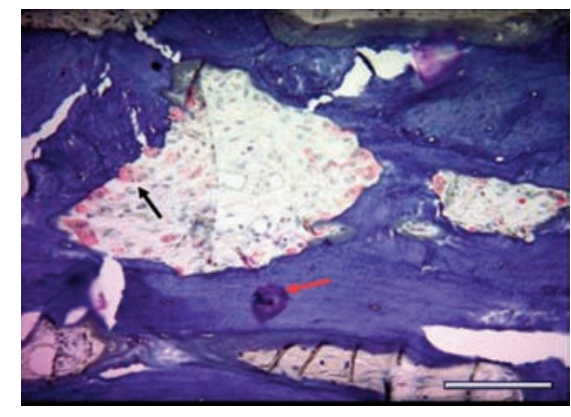

Figure 3. TRACP staining, incubation at $80^{\circ} \mathrm{C}$. Stained osteoclasts with decolorized areas (black arrow) and artefacts (red arrow). Scale bar: $4 \mu \mathrm{m}$. fixed the time of the reaction (in 30 minutes) while optimizing the temperature of the process.

Also, comparing to the method presented by Evans et al. ${ }^{15}$ there is a shortening of the reaction time by increasing the temperature.

The reason why higher temperatures give better and faster results is unclear and difficult to explain, but the effects of the processing and type of methacrylate used in enzymes may explain it.

Given the small number of activated osteoclasts in bone biopsies of patients with normal bone and, especially, in patients with adynamic bone disease (which now represent about $40 \%$ of the dialyzed population), the identification of TRACP expressed by these cells is crucial for qualitative and quantitative diagnosis. Standardizing the histochemistry reaction and optimizing the bright red uniform staining, (but keeping intact the cellular structure of osteoclasts) is particularly relevant when making bone histomorphometry, either by manual method or semi-automated method. ${ }^{22}$

According to our results, we recommended that for a time of $30 \mathrm{~min}$, incubation should be carried out at $60^{\circ} \mathrm{C}$. At this temperature the staining, identification and possible quantification of osteoclasts, based on TRACP expression, are optimized.

\section{References}

1. Ferreira MA. Biochemical markers of bone turnover in the diagnosis of renal osteodistrophy: what do we have, what do we need? Nephrol Dial Transplant 1998;13 29-32.

2. Ferreira MA. Diagnosis of renal osteodystrophy: when and how to use biochemical markers and non-invasive methods; when one biopsy is needed. Nephrol Dial Transplant 2000;15:8-14.

3. Malluche HH, Faugere MC. Atlas of mineralized bone histology. 1986, Karger Publ., Basel, Switzerland.

4. Moe S, Drueke T, Cunningham J, Goodman W, Martin K, Olgaard K, et al. Definition, evaluation, and classification of renal osteodystrophy: a position statement from Kidney Disease: Improving Global Outcomes (KDIGO). Kidney Int. 2006;69:1945-53.

5. Kaija H, Patrikainem L, Alatalo SL, Väänänen HK, Vihko PT. Acid Phosphatases. In: Seibel M, Robins S and Bilezikian J (eds) Dynamics of Bone and Cartilage Metabolism. 2nd ed, 2006. Academic Press, USA.

6. Vincent JB, Crowder MW, Averill BA. Spectroscopic and kinetics studies of a 
high-salt-stabilized form of the purple acid phosphatase from bovine spleen. Biochemistry 1991;30:3025-34.

7. Halleen JM, Räisänen S, Salo JJ, Reddy SV, Roodman GD, Hentunen TA, et al. Intracellular fragmentation of bone resorption products by reactive oxygen species generated by osteoclastic tartrateresistant acid phosphatase. J Biol Chem. 1999; 274:22907-10.

8. Ries WL, Key LL, Rogriguiz RM. Nitroblue tetrazolium reduction and bone resorption by osteoclasts in vitro inhibited by a manganese-based superoxide dismutase mimic. J Bone Miner Res 1992;7:931-9.

9. Beck JL, Durack MC, Hamilton SE, de Jersey J. Irreversible inactivation of purple acid phosphatase by hydrogen peroxide and ascorbate. J Inorg Biochem 1999;73: 245-52.

10. Ek-Rylander B, Flores M, Wendel M, Heinegård D, Andersson G. Dephosphorylation of osteopontin and bone sialoprotein by osteoclastic tartrate-resistant acid phosphatase. J Biol Chem 1994;269:148536.

11. Blair HC. How the osteoclast degrades bone. Bioessays 1998;20:837-46.
12. Hayman AR, Jones SJ, Boyde A, Foster D, Colledge WH, Carlton MB, et al. Mice lacking tartrate-resistant acid phosphatase (Acp 5) have disrupted endochondral ossification and mild osteopetrosis. Development 1996;122:3151-62.

13. Angel NZ, Walsh N, Forwood MR, Ostrowski MC, Cassady AI, Hume DA. Transgenic mice overexpressing tartrateresistant acid phosphatase exhibit an increased rate of bone turnover. $J$ Bone Miner Res 2000;15:103-10.

14. Bancroft J. Enzyme histochemistry. In: Bancroft $J$ and Stevens A (eds). Theory and practice of histological techniques. 2nd ed. 1982 Churchill Livingstone, London, UK.

15. Evans RA, Dunstan CR, Baylink DJ. Histochemical identification of osteoclasts in undecalcified sections of human bone. Miner Electrolyte Metab 1979;2:179-85.

16. Minkin C. Bone acid phosphatase: Tartrate-resistant acid phosphatase as a marker of osteoclast function. Calcif Tissue Int 1982;34:285-90.

17. Hayman AR, Warburton MJ, Pringle JA, Coles B, Chambers TJ. Purification and characterization of a tartrate-resistant acid phosphatase from human osteoclastomas. Biochem J 1989;261:601-9.

18. Dunstan CR, Evans RA. Quantitative bone histology: a new method. Pathology 1980;12:255-64.

19. Figueras SS, Barba MM. Bases histológicas de la histomorfometria ósea. In: Serrano S, Aubia J and Marinoso M (eds.) Patologia osea metabólica. 1990, Sandoz, Barcelona, Spain.

20. Malluche HH, Ritz E, Lange HP, Kutschera J, Hodgson M, Seiffert U, et al. Bone histology in incipient and advanced renal failure. Kidney Int 1976;9:355-62.

21. Galvão MJ, Ferreira A, Carvalho F, Costa AM. Técnica da biopsia óssea de osso não descalcificado com histomorfometria utilidade na caracterização da osteodistrofia renal. Rev Port Imunohistoquímica 1996;7:11-2.

22. Ferreira A, Carvalho F, Coelho A, Felicissimo P, Galvão MJ, Marques da Costa A. A utilidade da biopsia óssea com histomorfometria na caracterização da osteodistrofia renal. Rev Port Nefrol Hipert 1993;7:69-82. 